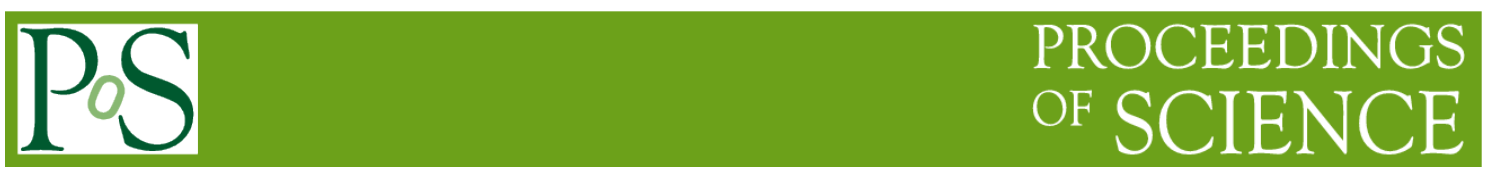

\title{
Status of the LHC and HL-LHC
}

\author{
Frederick Bordry ${ }^{1}$ \\ CERN \\ Geneva, Switzerland \\ E-mail: frederick.bordry@cern.ch
}

After two years of intense work of consolidation and upgrading of the LHC and injector performance, in 2015 the LHC went back into operation. The work program of the last two years is reviewed and the performance of the upgraded accelerator, including first collisions, is presented. A projection for the expected LHC performance in 2015 is given. Finally, an outlook of the future LHC upgrade programme at CERN, including the high-luminosity LHC, is given.

The European Physical Society Conference on High Energy Physics

22-29 July 2015

Vienna, Austria

${ }^{1}$ Speaker 